\title{
DESCRIPCIONES DE LOS ESTADOS INMADUROS DE CUATRO ESPECIES DE PHYLLOPHAGA, PARANOMALA Y MACRODACTYLUS (COLEOPTERA: MELOLONTHIDAE) DE LOS ALTOS DE CHIAPAS, MÉXICO
}

\author{
Concepción Ramírez-Salinas, ${ }^{1}$ Miguel Ángel Morón ${ }^{2}$ y Adriana E. \\ Castro-Ramírez ${ }^{1}$ \\ ${ }^{1}$ El Colegio de La Frontera Sur (ECOSUR). Carretera Panamericana y Periférico Sur s/n. San Cristóbal \\ de Las Casas, Chiapas, 29290 México. \\ ${ }^{2}$ Instituto de Ecología, A.C. Apartado Postal 63 Xalapa, Veracruz, 91000. México.<miguel.moron@ \\ inecol.edu.mx>
}

Ramírez-Salinas, C., M. Á. Morón \& A. E. Castro-Ramírez. 2011. Descripciones de los estados inmaduros de cuatro especies de Phyllophaga, Paranomala y Macrodactylus (Coleoptera: Melolonthidae) de Los Altos de Chiapas, México. Acta Zoológica Mexicana (n. s.), 27(3): 527-545.

RESUMEN. Se describen las larvas de tercer estadio de Phyllophaga pilosula (Moser), Paranomala denticollis (Bates), Paranomala trapezifera (Bates) y Macrodactylus fulvescens Bates, y la pupa de la última especie, procedentes de San Cristóbal de Las Casas, Amatenango del Valle y Yalumá-Villahermosa, municipio de Comitán, Chiapas, México. Las larvas de las especies de Phyllophaga y Paranomala se obtuvieron a partir de la cría desde huevos depositados por hembras en cautiverio. Las larvas de tercer estadio de M. fulvescens fueron recolectadas en suelo con cultivo de maíz hasta obtener exuvias, pupas y adultos. Se incluyen ilustraciones de las estructuras características y comentarios para distinguirlas de especies afines locales.

Palabras Clave: "gallina ciega", Melolonthinae, Rutelinae, pupas, larvas saprófagas, suelos agrícolas, maíz.

Ramírez-Salinas, C., M. Á. Morón \& A. E. Castro-Ramírez. 2011. Descriptions of the immature stages of four species of Phyllophaga, Paranomala and Macrodactylus (Coleoptera: Melolonthidae) from Los Altos de Chiapas, Mexico. Acta Zoológica Mexicana (n. s.), 27(3): 527-545.

ABSTRACT. Third instar larvae of Phyllophaga pilosula (Moser), Paranomala denticollis (Bates), Paranomala trapezifera (Bates) and Macrodactylus fulvescens Bates and the pupa of the latter species, are described with specimens from San Cristobal de Las Casas, Amatenango del Valle and Yalumá-Villahermosa in Comitan, Chiapas, Mexico. Adults of Phyllophaga and Paranomala species were captured and reared in captivity to obtain third-stage larvae. Through the development of third stage larvae of M. fulvescens collected from soil with corn crops, were obtained exuvia of third instar larva, pupae and

Recibido: 15/06/2010; aceptado: 18/05/2011. 
adults. Drawings of the diagnostic structures and brief comments on the differences with local related species are included,

Keywords: White grubs, Melolonthinae, Rutelinae, pupae, saprophagous larvae, agricultural soils, corn.

\section{INTRODUCCIÓN}

Aún se conoce poco sobre la identidad de las larvas escarabeiformes presentes en las parcelas agrícolas, pero puede estar presente un complejo de géneros y especies con distintos hábitos alimentarios; esto trae como consecuencia que los métodos de control, sobre todo el químico, para las que consumen raíces y son consideradas plaga, afecten también a las larvas saprófagas y facultativas (Ramírez-Salinas et al. 2001). En diversos estudios realizados en el cultivo de maíz de los Altos de Chiapas, se ha comprobado que el complejo "gallina ciega" está formado por más de 12 especies de Melolonthidae, como las de hábitos rizófagos estrictos: Phyllophaga menetriesi (Blanchard, 1850), Phyllophaga tenuipilis (Bates, 1888), Phyllophaga ravida (Blanchard, 1850), Phyllophaga obsoleta (Blanchard, 1850) y Phyllophaga testaceipennis (Blanchard, 1850); las de hábitos rizófagos facultativos: Phyllophaga tumulosa (Bates, 1888), Paranomala sticticoptera (Blanchard, 1850) y Paranomala inconstans (Burmeister, 1844); y las predominantemente saprófagas: Paranomala trapezifera (Bates, 1888), Paranomala denticollis (Bates, 1888), Hoplia spp., Macrodactylus spp. y Diplotaxis spp. (Castro-Ramírez et al. 2004, 2005).

En varias localidades de Chiapas se han recolectado adultos de Phyllophaga pilosula (Moser), Paranomala trapezifera, Paranomala denticollis y Macrodactylus fulvescens. Los adultos de Phyllophaga pilosula, de hábitos nocturnos, se han capturado en áreas agrícolas cercanas a bosques en Aguacatenango, Navil, Pacvilná, Piedra Escrita, Las Piedrecitas, San Cristóbal, Tenejapa, Tzunum y Winikton, pero no se han encontrado sus larvas en suelos de parcelas agrícolas, por lo que se desconocen sus características y preferencias alimenticias. Los adultos de Paranomala trapezifera se han recolectado cerca de las áreas agrícolas de Aguacatenango, Navil, San Cristóbal, Piedra Escrita, Tenejapa y Tzunum, pero no se tienen datos de sus larvas. Los adultos de Paranomala denticollis se capturaron en Aguacatenango, El Madronal, Pacvilná, Piedra Escrita, Teopisca, Tzunum, Winikton y Yalumá Villahermosa, son de hábitos nocturnos (Castro Ramírez et al. 2004, 2005). Las larvas y adultos de Macrodactylus fulvescens se han encontrado en las parcelas con maíz de Yalumá Villahermosa, municipio de Comitán, pero se desconocen sus hábitos alimentarios.

Sólo se conoce el 1\% de las especies de "gallina ciega" que habitan en los suelos cultivados de América Latina (Ramírez-Salinas et al. 2000), y para desarrollar un manejo integrado de plagas subterráneas es indispensable la identificación precisa de las especies asociadas al cultivo, el estudio de sus hábitos y ciclos de vida (Morón et al. 1997). Por ello, en este trabajo se presentan las descripciones de las larvas de 
tercer estadio de Ph. pilosula, $P$. denticollis, $P$. trapezifera y de la larva y la pupa de M. fulvescens.

\section{MATERIALES Y MÉTODOS}

Los adultos de Ph. pilosula y P. trapezifera fueron recolectados en mayo de 2005 y 2006 en San Cristóbal de Las Casas, los de P. denticollis el 4 de mayo de 2006 en Amatenango del Valle. Fueron transportados al laboratorio de bioensayos de El Colegio de la Frontera Sur (ECOSUR) donde se determinaron y separaron por parejas en recipientes con $1 \mathrm{~kg}$ de suelo húmedo tapados con tela de tul y adecuadamente etiquetados, para esperar la oviposición y el desarrollo de los primeros dos estadios larvales. Cuando se obtuvieron las larvas de tercer estadio se fijaron en solución de Pampel y una semana después se pasaron a alcohol al 70\% para su conservación. Las larvas de M. fulvescens se obtuvieron en muestreos de suelo de parcelas con maíz en Yalumá Villahermosa, municipio de Comitán, el 10 y 16 se septiembre de 2001, las larvas se depositaron individualmente en recipientes con $40 \mathrm{~g}$ de suelo húmedo. La mitad de la muestra se fijó en solución de Pampel y luego se conservó en alcohol etílico al 70\%, la otra mitad se dejó desarrollar para obtener exuvias, pupas y adultos.

Las observaciones e ilustraciones se hicieron con un estereomicroscopio Carl Zeiss SV6 usando los aumentos de 1.6 a $5 \mathrm{X}$; las mediciones se realizaron con un estereomicroscopio Nikon SMZ-U zoom 1:10 con aumentos de 1 a 7.5X. Los caracteres y la terminología utilizada en las descripciones son los propuestos por Boving (1942), Ritcher (1966) y Morón (1986). Los ejemplares estudiados están depositados en la colección del proyecto "Diversidad en Sistemas de Cultivos" de ECOSUR, San Cristóbal de Las Casas, Chiapas, y en la Colección Entomológica IEXA, INECOL, Xalapa, Veracruz.

\section{RESULTADOS}

\section{Phyllophaga (Phyllophaga) pilosula (Moser, 1918)}

Larva de tercer estadio (Figs. 1-14).

Descripción $(\mathrm{N}=5)$. Cabeza (Fig. 1) Anchura máxima de la cápsula cefálica 4.50$4.85 \mathrm{~mm}$. Superficie del cranium casi lisa, parda amarillenta. Frente con 1 seda frontal exterior y 1 seda frontal posterior a cada lado; 13-15 sedas frontales anteriores; con 1 seda en los ángulos anteriores a la frente; el resto de la superficie craneal con 1-2 sedas dorso-epicraneales, con 1 seda epicraneal y 6-9 sedas paraocelares a cada lado. Clípeo con 2 sedas centrales y 1-2 sedas laterales a cada lado. Labro simétrico, con los márgenes laterales redondeados, 8 a 14 sedas posteriores, 2 sedas centrales y 3 sedas laterales a cada lado. Manchas oculares ausentes. Epifaringe (Fig. 2) con 2.02- 
$2.20 \mathrm{~mm}$ de anchura y $1.47-1.70 \mathrm{~mm}$ de longitud, sin zygum y con epizygum estrecho, irregular, pardo oscuro; haptomerum prominente con 6-8 heli; cada plegmatium formado con 9-11 plegmata anchos. Cada acanthoparia con 9-12 sedas espiniformes recurvadas. Proplegmatia ausente. Dexiophoba irregular y fragmentada y laeophoba grande, extendida a través del borde de la laeotorma. Dexiotorma casi recta, con los extremos redondeados; laeotorma corta. Haptolachus con algunas microsensilas dispersas y un cono sensorial redondeado. Placa esclerosada grande y angulosa. Crepis bien diferenciado. Chaetoparia izquierda con 41 a 61 sedas gruesas y 22 a 34 sedas delgadas. Chaetoparia derecha 31 a 42 sedas gruesas y 16 a 30 sedas delgadas. Mandíbulas (Figs. 3-4) sin área estriduladora ventral, áreas incisivas de ambas mandíbulas con una lámina cortante distal separada de un dientecillo incisivo proximal por una escotadura incisiva; márgenes internos sin dientes; área molar de la mandíbula izquierda con el lóbulo distal (M1) bien desarrollado; brustia moderadamente setosa; área molar de la mandíbula derecha con el lóbulo distal poco desarrollado; calx prominente y brustia moderadamente setosa. Maxilas (Figs. 5, 8) con un uncus terminal en la galea y tres unci alineados en la lacinia, con 4-5 heli a cada lado. Área estriduladora maxilar con 9-15 dientecillos. Escleroma hipofaríngeo (Fig. 9) asimétrico, con el lado derecho prominente. Último artejo antenal con un área sensorial ovalada dorsal (Fig. 6) y dos áreas sensoriales ventrales (Fig. 7).

Tórax. Los estigmas respiratorios (Fig. 13) miden 0.39-0.44 $\mathrm{mm}$ de largo por 0.53-0.63 mm de ancho; placa respiratoria amarillo rojiza, uniformemente recurvados en forma de "C", la distancia entre los lóbulos de la placa respiratoria es menor que el diámetro dorso ventral de la bulla redondeada. Pronoto con un escleroma lateral alargado a cada lado, amarillo cremoso, con sedas largas. Proprescudo con 9-12 sedas cortas y largas distribuidas de forma irregular; proescudo con 6-10 sedas cortas y largas irregularmente situadas; mesoprescudo con 5 a 7 sedas medianas y largas en hilera transversal; mesoescudo sin sedas; mesoescutelo con 5 a 7 sedas medianas y largas en hilera; metaprescudo con 4 a 6 sedas cortas y largas en hilera; metaescudo sin sedas; metaescutelo con 5 a 8 sedas cortas y largas en hilera transversal. Uñas pro y mesotarsales largas, con el ápice afilado, con una seda prebasal interna y una lateral externa (Figs. 10-11); uñas metatarsales afiladas, más cortas y estrechas que las precedentes (Fig. 12).

Abdomen. Estigmas de los segmentos I a VII casi semejantes en diámetro, 0.15$0.41 \mathrm{~mm}$ de largo por $0.27-0.47 \mathrm{~mm}$ de ancho; placa respiratoria amarillo rojiza, regularmente recurvada en forma de "C". Dorsa de los segmentos abdominales I-VI con numerosas sedas espiniformes y pocas sedas largas distribuidas transversalmente, segmento VII con algunas sedas espiniformes y sedas largas; segmentos VIII a IX sin sedas espiniformes, con sedas largas transversales. Venter de los segmentos de I a IX con 7 a 12 sedas cortas y largas distribuidas transversalmente. Raster con un par de palidia longitudinales ligeramente convergentes en ambos extremos, con una 


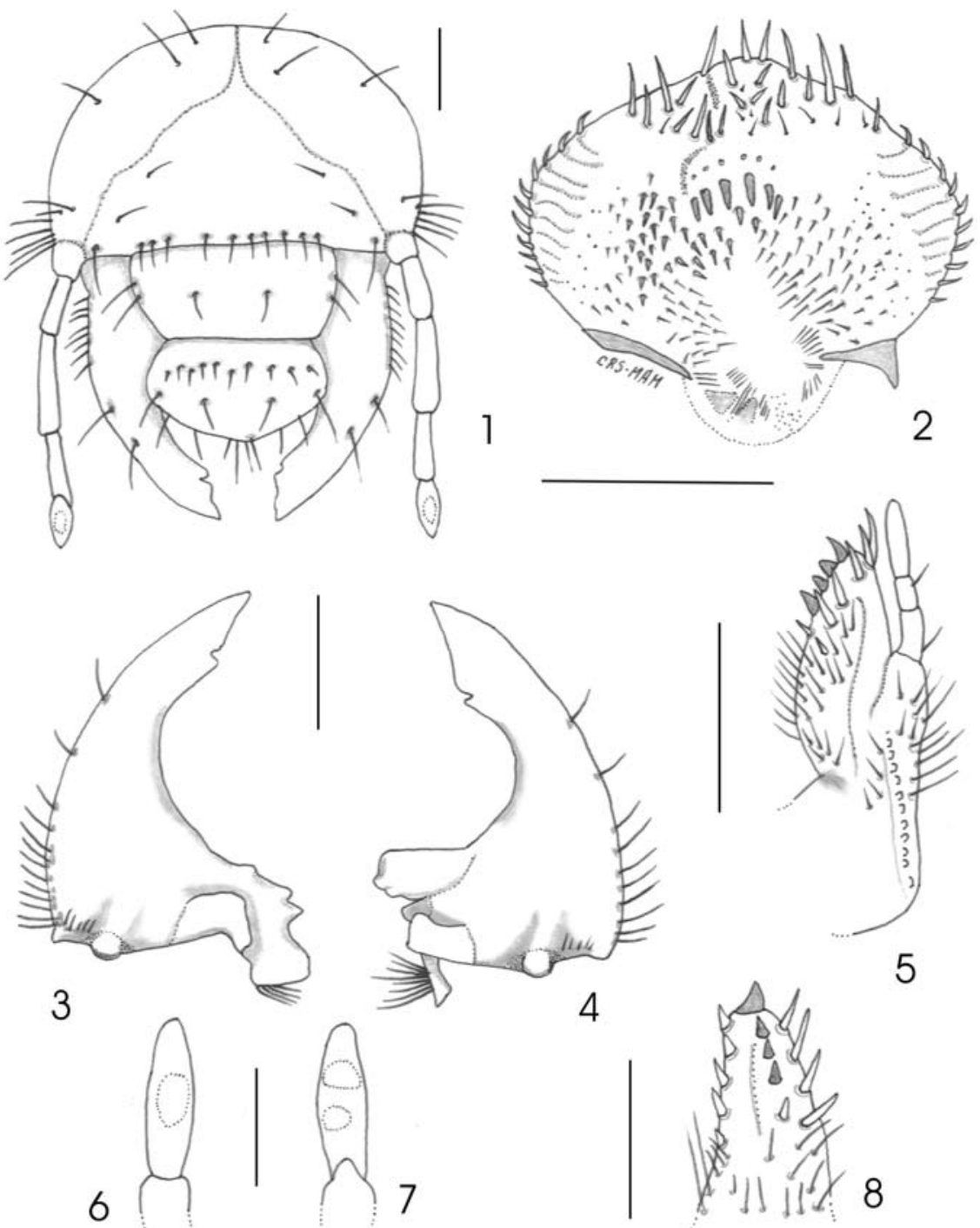

Figuras 1-8. Larva de tercer estadio de Phyllophaga pilosula. 1. Vista frontal de la cabeza. 2. Aspecto ventral de la epifaringe. Mandíbulas en vista ventral: 3. izquierda. 4. derecha. 5. Maxila derecha, dorsal. Último artejo antenal: 6 . dorsal. 7. ventral. 8. Vista mesial del ápice de la maxila derecha. Líneas de escala $=1 \mathrm{~mm}$, excepto figs. $6-8=0.5 \mathrm{~mm}$.

longitud de 1.4-1.8 mm, que definen una septula estrecha y se encuentran separadas en su parte central por espacio de 0.4-0.6 mm (Fig. 14); cada palidium está formado por 13-21 pali. Tegilla con 25 a 33 sedas hamate, que generalmente no rodean los 

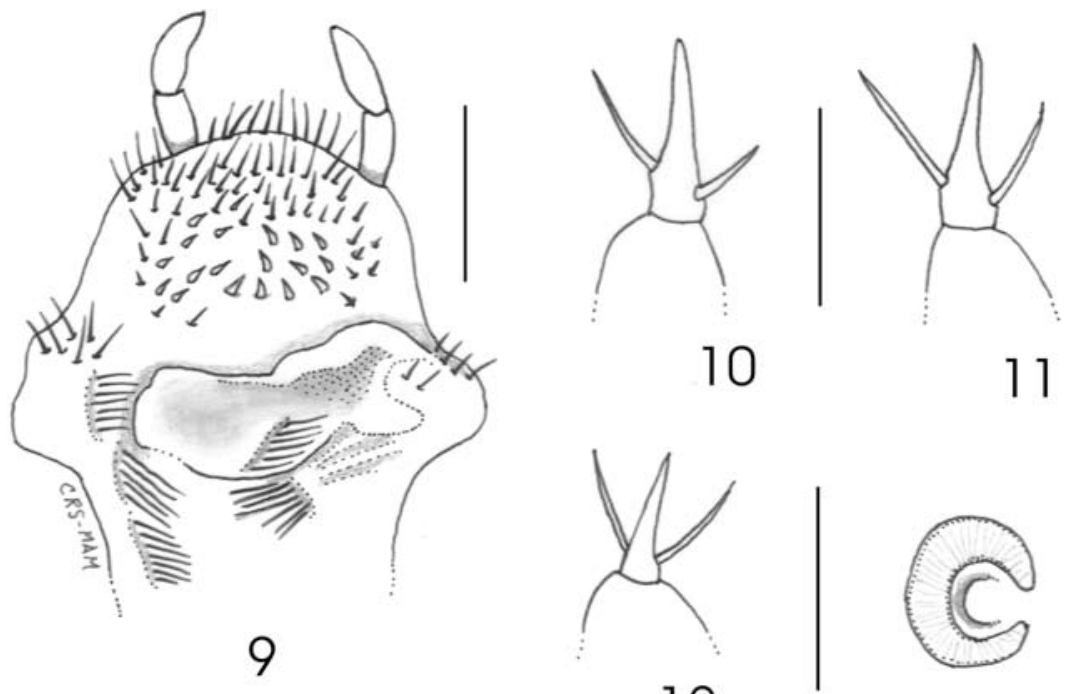

12

13

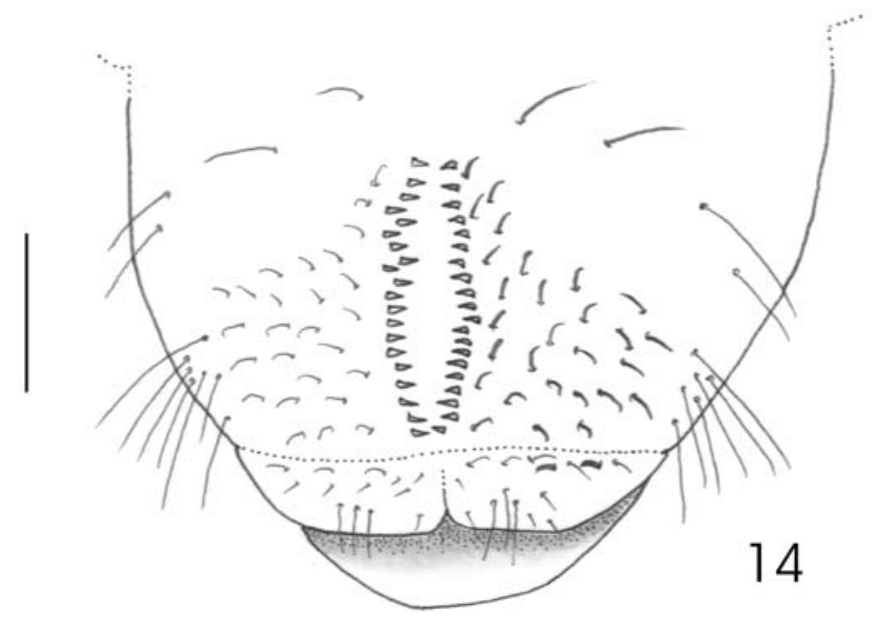

Figuras 9-14. Larva de tercer estadio de Phyllophaga pilosula. 9. Hipofaringe. Uñas: 10. protarsal. 11. mesotarsal. 12. metatarsal. 13. Estigma respiratorio protorácico. 14. Raster. Líneas de escala $=0.5 \mathrm{~mm}$, excepto en fig. $14=1 \mathrm{~mm}$.

extremos anteriores de las palidia. Campus con 9 sedas largas y finas. Barbula moderadamente setosa. Labio anal dorsal con 7-9 sedas hamate, con sedas espiniformes cortas y algunas sedas largas. Labio anal ventral claramente escotado. Abertura anal en forma de "Y". Longitud total aproximada del cuerpo $3.62 \mathrm{~cm}$ en promedio, pero puede medir de 2.7 a $4.2 \mathrm{~cm}$. 
Material examinado. Cinco larvas de tercer estadio obtenidas en el laboratorio el 2 y 8-VIII-2005,15-IX-2005 y 18-IX-2006, a partir de adultos recolectados en México: Estado de Chiapas, municipio San Cristóbal de Las Casas, el 17-V-05 y 10-V-06. J. Carmona de la Torre.

Comentarios. La larva de Ph. pilosula es parecida a la de Ph. obsoleta, la primera se diferencia por registrar de 13-15 sedas frontales anteriores, haptomerum con 6-8 heli, las sedas hamate no rodean los extremos anteriores de la palidia, cada palidium está formado por 13-21 pali. Las larvas de P. obsoleta presentan de 8-13 sedas frontales anteriores, haptomerum con 8-10 heli, las sedas hamate rodean los extremos anteriores a la palidia, cada palidium está formado por 15 a 27 pali (Ramírez-Salinas et al. 2000).

\section{Paranomala denticollis (Bates, 1888)}

Larva de tercer estadio (Figs. 15-28)

Descripción ( $\mathrm{N}=5)$. Cabeza (Fig. 15). Anchura máxima de la cápsula cefálica 2.40$2.75 \mathrm{~mm}$. Superficie del cranium amarillo claro, lisa. Frente carece de sedas frontales exteriores y tiene una seda frontal posterior a cada lado; 2 sedas frontales anteriores; 2 sedas en los ángulos anteriores de la frente; con 4-5 sedas dorso epicraneales, 1-2 sedas epicraneales y 4-5 sedas basiantenales a cada lado. Clípeo con 2 sedas centrales y 2-2 sedas laterales a cada lado. Labro ligeramente asimétrico, con 6 a 8 sedas posteriores, 2 sedas centrales y 3 sedas laterales a cada lado. Epifaringe (Fig.16) tiene de ancho de $1.13-1.17 \mathrm{~mm}$ y de longitud de $0.85-0.87 \mathrm{~mm}$; con epizygum alargado, irregular, pardo obscuro, haptomerum con una hilera transversal de 3 a 4 heli; cada plegmata formada por 9-14 plegmatia cortos, levemente visibles; acanthoparia con 9-13 sedas espiniformes recurvadas y cortas, levemente visibles; carece de proplegmatia, clithra; laeophoba escasa; dexiotorma casi recta y delgada. Placa esclerosada grande y angulosa, cono sensorial prominente. Crepis estrecho pero bien definido. Pedium amplio. Chaetoparia izquierda con 45-51 sedas gruesas y 22-28 sedas delgadas. Chaetoparia derecha con 41-48 sedas gruesas y de 18-24 sedas delgadas. Acroparia y corypha fusionados. Mandíbulas (Figs. 17-18) con área estriduladora ventral. Áreas incisivas de ambas mandíbulas con una lámina cortante distal separada de un dientecillo incisivo proximal por una escotadura incisiva; márgenes internos sin dientes; área molar de la mandíbula izquierda con el lóbulo distal (M1) bien desarrollado; acia poco prominente, redondeada, brustia levemente setosa; área molar de la mandíbula derecha con 3 rebordes bien diferenciados; calx prominente y brustia levemente setosa. Maxilas (Figs.19-20) con 1 uncus en la galea, 2 unci alineados en lacinia, con hileras de 2-3 heli a cada lado. Área estriduladora maxilar formada con 6 a 7 dientecillos. Escleroma hipofaríngeo asimétrico (Fig. 23) con el lado derecho prominente y el izquierdo reducido. Último artejo antenal (Figs. 21-22) con 1 área sensorial ovalada en la superficie dorsal y 1-2 áreas sensoriales en la superficie ventral. 

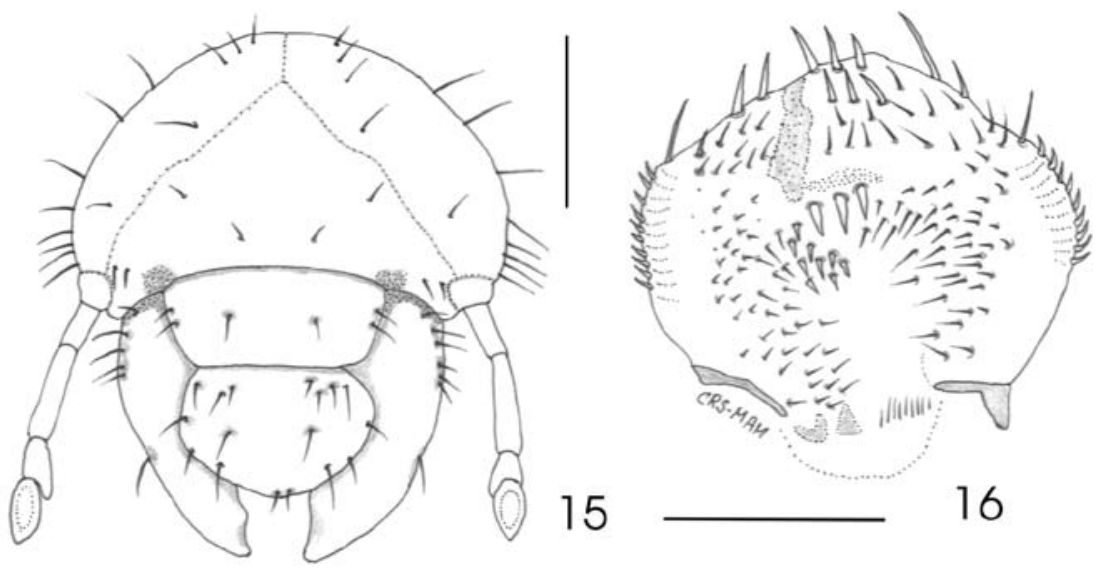

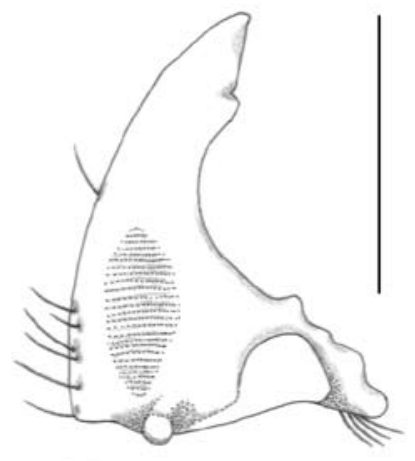

17

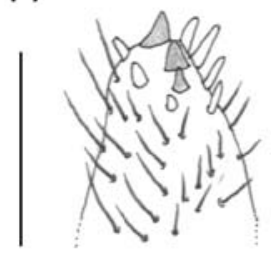

20

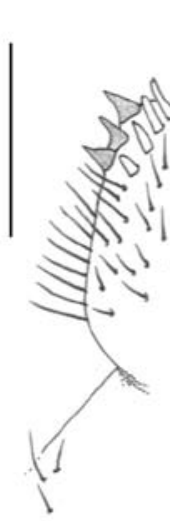

18

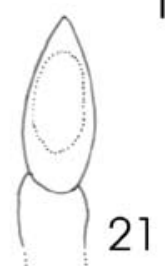

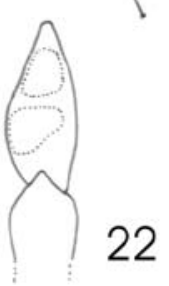

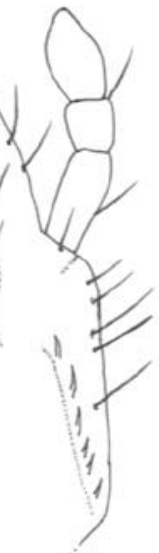

19

Figuras 15-22. Larva de tercer estadio de Paranomala denticollis. 15. Vista frontal de la cabeza.

16. Aspecto ventral de la epifaringe. Mandíbulas en vista ventral: 17. izquierda. 18. derecha.

19. Maxila derecha, dorsal. 20. Vista mesial del ápice de la maxila derecha. Último artejo antenal:

21. dorsal. 22. ventral. Líneas de escala $=1 \mathrm{~mm}$, excepto figs. $19-22=0.5 \mathrm{~mm}$.

Tórax. Pronoto con un escleroma lateral corto a cada lado, amarillo ámbar, con 4 a 7 sedas medianas y largas. Los estigmas respiratorios (Fig. 27) miden 0.16-0.20 $\mathrm{mm}$ de largo por $0.25-0.27 \mathrm{~mm}$ de ancho; placa respiratoria amarilla, uniformemente recurvado en forma de "C", la distancia entre los lóbulos de la placa respiratoria es 
menor que el diámetro dorso ventral de la bulla que es redondeada y poco visible. Proprescudo con 4-7 sedas cortas, medianas y largas distribuidas irregularmente; proescudo con 6-11 sedas medianas y largas distribuidas de manera irregular; mesoprescudo con 9-11 sedas medianas y largas en sentido transversal; mesoescudo sin sedas; mesoescutelo con 7-11 sedas medianas y largas en hilera transversal; metaprescudo con 10-12 sedas medianas y largas en hilera transversal; metaescudo sin sedas; metaescutelo con 14-18 sedas medianas y largas en hilera transversal. Uñas pro y mesotarsales alargadas, con ápice agudo, y una seda interior prebasal y una seda exterior intermedia (Figs. 24-25); uña metatarsal afilada pero un poco más corta que las precedentes (Fig. 26).

Abdomen. Estigmas de los segmentos de I a VIII casi semejantes en diámetro, $0.12-0.21 \mathrm{~mm}$ de largo por $0.15-0.25 \mathrm{~mm}$ de ancho; placa respiratoria amarilla, regularmente recurvada en forma de "C". Dorsa de los segmentos abdominales de I a VI con numerosas sedas espiniformes y algunas sedas largas distribuidas en sentido transversal; el VII segmento con 2 hileras de sedas largas y algunas sedas espiniformes, los segmentos VIII y IX con 2 hileras transversas de sedas medianas y largas. Venter de los segmentos I a IX con 7-15 sedas medianas y largas transversalmente. Raster (Fig. 28) con un par de palidia longitudinales y paralelas, siendo más amplio hacia el labio anal inferior, con una longitud de $0.44-0.53 \mathrm{~mm}$ y se encuentran separados en su parte superior por $0.12-0.17 \mathrm{~mm}$ y en la parte inferior por $0.20-0.32 \mathrm{~mm}$; cada palidium está formada por 11-13 pali espiniformes cortos. Tegilla formadas con 38-50 sedas hamate cerca de las palidia y en los extremos anteriores de éstas. Labio anal ventral bien diferenciado con 16-19 sedas hamate y sedas amarillas largas. Campus con 9-10 sedas largas. Barbula ligeramente setosa y amarillenta. Abertura anal transversal, ligeramente recurvada. La longitud dorsal aproximada del cuerpo es de 2.5 a $3.0 \mathrm{~cm}$.

Material examinado. Cinco larvas de tercer estadio obtenidas en laboratorio el 18IX-2006 y 16-X-2006, a partir de adultos recolectados en México: Estado de Chiapas, municipio Amatenango del Valle, 4-V-06. C. Ramírez-Salinas.

Comentarios. Las larvas de $P$. denticollis se diferencian de $P$. inconstans porque las primeras tienen una seda frontal posterior a cada lado, un par de palidia longitudinales y paralelas, más separadas entre sí hacia el labio anal inferior, con una longitud de 0.44-0.53 mm; cada palidium formada por 11-13 pali espiniformes, tegilla formada por 38-50 sedas hamate que rodean a la palidia, labio anal ventral con 16-19 sedas hamate. De acuerdo con Ramírez-Salinas et al. (2004) las larvas de P. inconstans presentan 2 sedas frontales posteriores, un par de palidia longitudinales, paralelas y ligeramente divergentes hacia el labio anal inferior, con una longitud de 1.10-1.35 $\mathrm{mm}$, cada palidium formado por 11-17 pali espiniformes, tegilla formadas con 62-77 sedas hamate, labio anal ventral con 31 a 45 sedas hamate. 

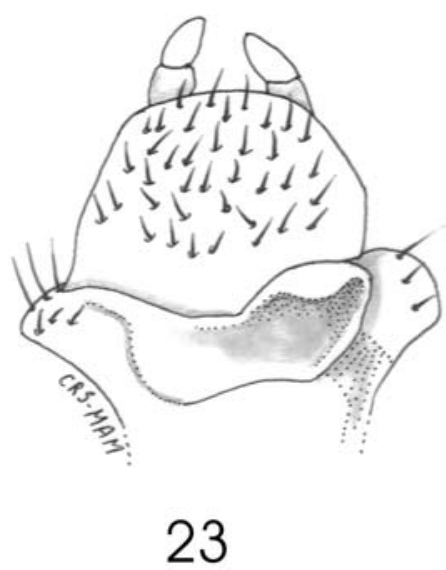
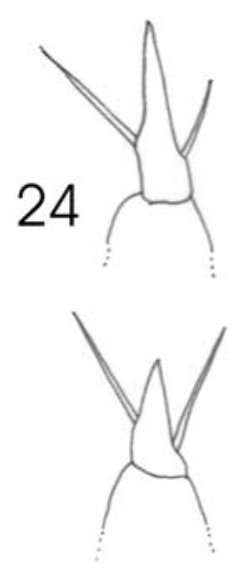

26

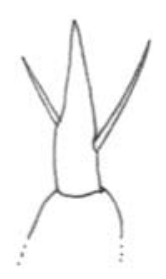

25

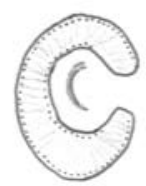

27

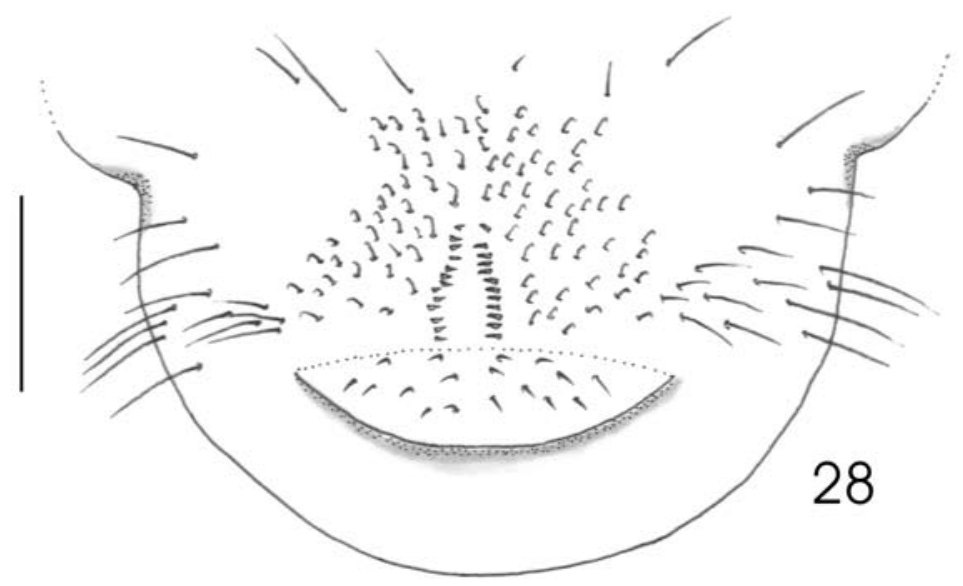

Figuras 23-28. Larva de tercer estadio de Paranoma denticollis. 23. Hipofaringe. Uñas: 24. protarsal. 25. mesotarsal. 26. metatarsal. 27. Estigma respiratorio protorácico. 28. Raster. Líneas de escala $=0.5$ $\mathrm{mm}$, excepto en fig. $28=1 \mathrm{~mm}$.

\section{Paranomala trapezifera (Bates, 1888)}

Larva de tercer estadio (Figs. 29-42)

Descripción ( $\mathrm{N}=2$ ). Cabeza (Fig. 29). Anchura máxima de la cápsula cefálica: 3.40$3.55 \mathrm{~mm}$. Superficie del cranium parda amarillenta, lisa. Frente con 1 seda frontal exterior y 2 sedas frontales posteriores a cada lado; 2 sedas frontales anteriores; $2-3$ sedas en los ángulos anteriores de la frente; 2 sedas epicraneales, 3-4 sedas dorso 
epicraneales y 5 sedas basiantenales a cada lado. Clípeo con 2 sedas centrales, 3-4 sedas laterales a cada lado. Labro ligeramente asimétrico, con 6-7 sedas posteriores, 2 sedas centrales y 3 sedas laterales a cada lado. Epifaringe (Fig. 30) tiene una anchura de $1.60 \mathrm{~mm}$ y una longitud de $1.12 \mathrm{~mm}$, con epizygum alargado, irregular, pardo obscuro, haptomerum con una hilera transversal de 3 heli, cada plegmata formada por 11 plegmatia cortos, levemente visibles; cada acanthoparia con 10-11 sedas espiniformes recurvadas y cortas, carece de proplegmatia y clithra; laeophoba escasa. Dexiotorma casi recta y delgada; placa esclerosada grande y angulosa, cono sensorial prominente. Crepis bien definido. Pedium amplio. Chaetoparia izquierda con 56 sedas gruesas y 35 sedas delgadas. Chaetoparia derecha con 43 sedas gruesas y 33 sedas delgadas. Mandíbulas (Figs. 31-32) con área estriduladora ventral. Áreas incisivas con una lámina cortante distal separada de un dientecillo incisivo proximal por una escotadura incisiva; márgenes internos de la mandíbula izquierda con un diente; área molar de la mandíbula izquierda con el lóbulo distal (M1) bien desarrollado; brustia moderadamente setosa; área molar de la mandíbula derecha con 3 rebordes bien definidos; calx prominente y brustia moderadamente setosa. Maxilas (Figs. 3334) con 1 uncus en galea y 2 unci alineados en lacinia, con hileras de 2-3 heli a cada lado. Área estriduladora maxilar formada de 7 dientecillos. Escleroma hipofaríngeo asimétrico (Fig. 37). Último artejo antenal (Figs. 35-36) con un área sensorial ovalada en la superficie dorsal y dos áreas sensoriales en la superficie ventral.

Tórax. Pronoto con un escleroma lateral corto a cada lado, amarillo claro, con 3 -4 sedas cortas y largas. Los estigmas respiratorios (Fig. 41) miden 0.25-0.27 mm de largo por $0.33-0.35 \mathrm{~mm}$ de ancho; placa respiratoria amarilla, regularmente recurvada en forma de "C", la distancia entre los lóbulos de la placa respiratoria es menor que el diámetro dorso ventral de la bulla redondeada. Proprescudo con 3 sedas medianas en hilera, proescudo con 9 sedas medianas y largas en hilera, mesoprescudo con 10 sedas medianas y largas en hilera irregular, mesoescudo sin sedas, mesoescutelo con 6 sedas medianas y largas en hilera, metaprescudo con 8 sedas medianas y largas en hilera, metaescudo sin sedas; metaescutelo con 10 sedas medianas y largas en hilera, más 4 sedas espiniformes. Uñas pro y mesotarsales (Figs. 38-39) alargadas, afiladas, con una seda externa a cada lado; y uñas metatarsales (Fig. 40) casi del mismo tamaño que las anteriores, pero con el extremo distal un poco recurvado.

Abdomen. Estigmas de los segmentos I-VIII casi semejantes en diámetro, 0.15$0.33 \mathrm{~mm}$ de largo por $0.27-0.37 \mathrm{~mm}$ de ancho; placa respiratoria amarilla, regularmente recurvada en forma de "C". Dorsa de los segmentos abdominales de I-VI con numerosas sedas espiniformes y algunas sedas largas distribuidas en sentido transversal; segmentos VII-IX sin sedas espiniformes con 2 hileras transversales de sedas medianas y largas. Raster (Fig. 42) con un par de palidia longitudinales, paralelas y amplio hacia el labio anal inferior, alcanzan una longitud de $0.69-0.71 \mathrm{~mm}$ y se encuentran separados en su parte superior $0.20 \mathrm{~mm}$ y en la parte inferior $0.35-0.41$ 

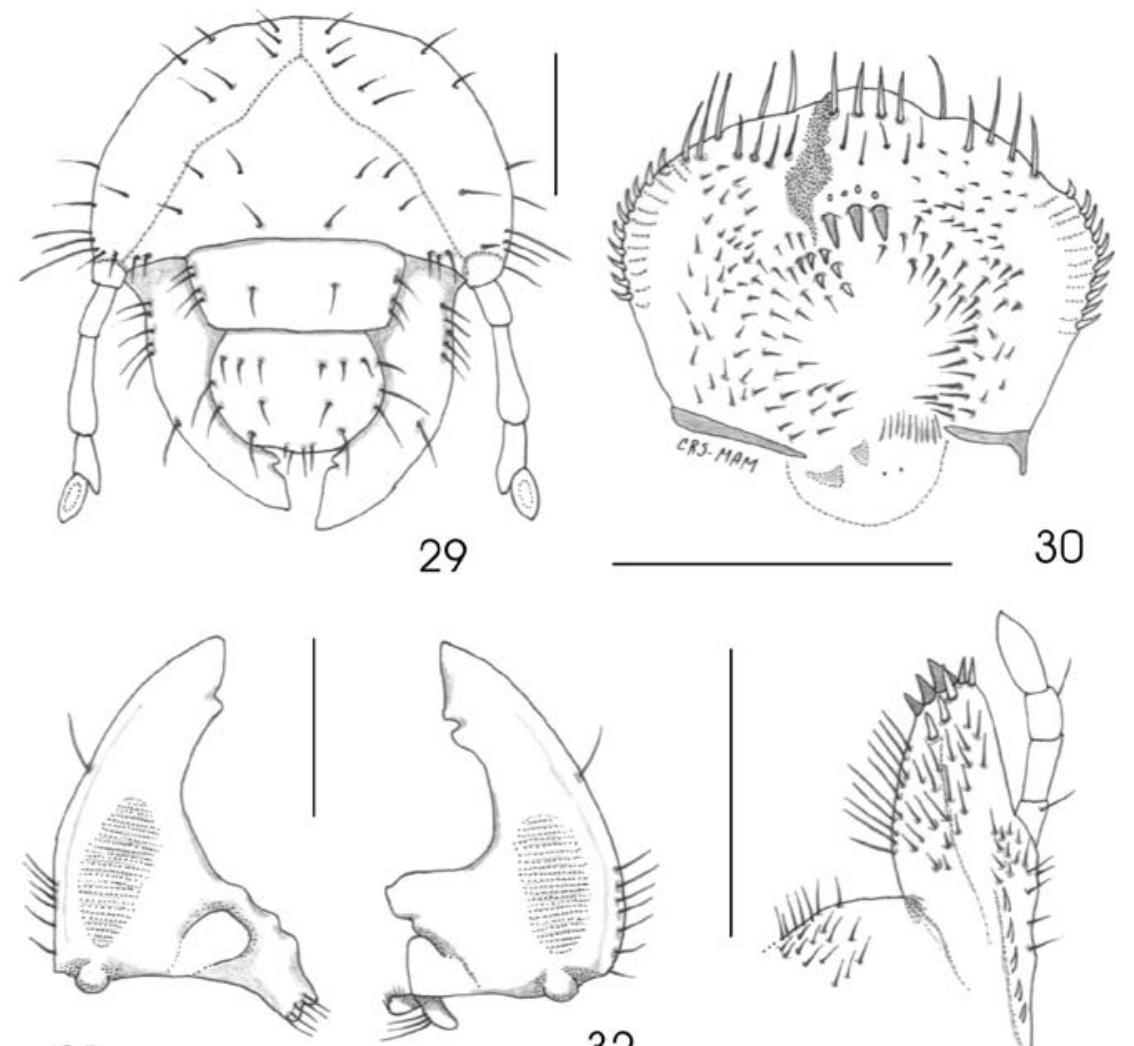

31
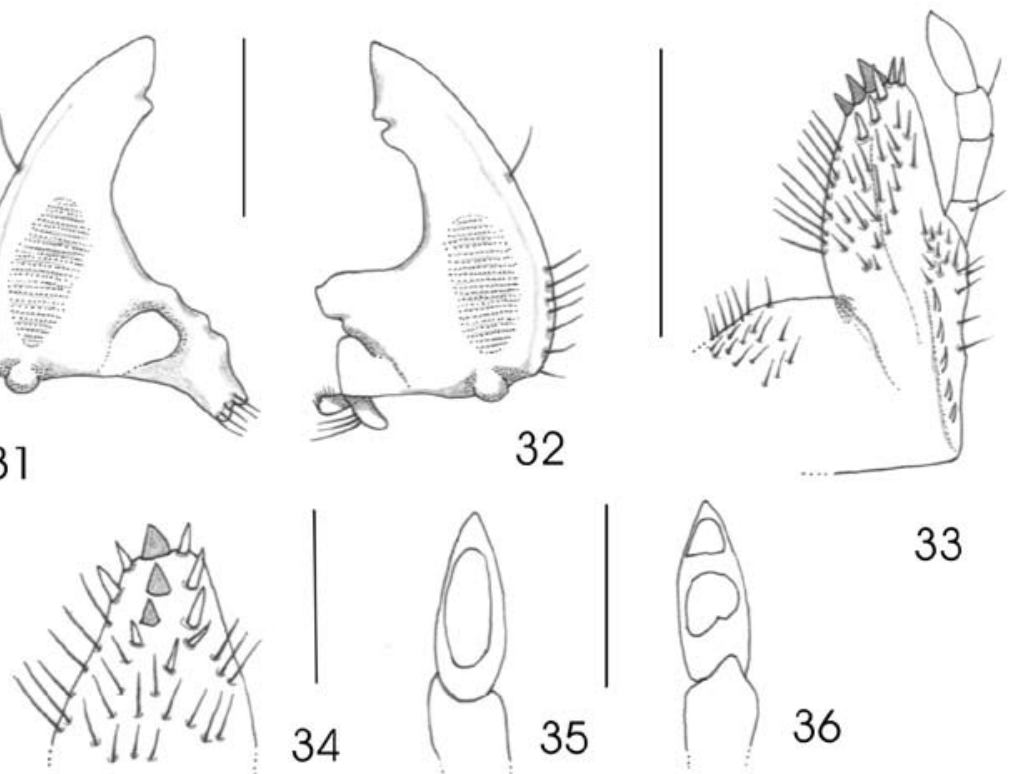

33

Figuras 29-36. Larva de tercer estadio de Paranomala trapezifera. 29. Vista frontal de la cabeza.

30. Aspecto ventral de la epifaringe. Mandíbulas en vista ventral: 31 . izquierda. 32. derecha. 33. Maxila derecha, dorsal. 34. Vista mesial del ápice de la maxila derecha. Último artejo antenal: 35. dorsal. 36. ventral. Líneas de escala $=1 \mathrm{~mm}$, excepto figs. $34-36=0.5 \mathrm{~mm}$.

mm, cada palidium está formado por 13-17 pali espiniformes. Tegilla formada por 32-35 sedas hamate cerca de la palidia y en los extremos anteriores a ésta. Labio anal ventral bien diferenciado con 23-29 sedas hamate y sedas largas amarillas. Campus con 10 sedas largas. Barbula ligeramente setosa y amarillenta. Abertura anal trans- 
versal, ligeramente recurvada. La longitud dorsal aproximada del cuerpo es de 2.3 a $3.0 \mathrm{~cm}$.

Material examinado. Dos larvas de tercer estadio obtenidas en el laboratorio 7-IX2005 y 16-X-2006, a partir de adultos recolectados en México: Estado de Chiapas, municipio San Cristóbal de Las Casas, el 17-V-2005 y 10-V-2006. C. Ramírez-Salinas y J. Carmona de La Torre.

Comentarios. Las larvas de $P$. trapezifera se diferencian de $P$. denticollis por tener una cápsula cefálica más grande, de 3.40-3.55 mm, con 1 seda frontal exterior, 2 sedas frontales posteriores, 2-3 sedas en los ángulos anteriores de la frente; un par de palidia longitudinales y paralelas, con una extensión de 0.69-0.71 mm. Las larvas de $P$. denticollis tienen una cápsula cefálica más pequeña, de $2.40-2.75 \mathrm{~mm}$, carecen de seda frontal exterior, con 1 seda frontal posterior, 2 sedas en los ángulos anteriores a la frente; con un par de palidia longitudinales y paralelas, con una extensión de $0.44-$ $0.53 \mathrm{~mm}$.

\section{Macrodactylus fulvescens Bates, 1887}

Larva de tercer estadio (Figs. 43-56)

Descripción $(\mathrm{N}=4)$. Cabeza (Fig. 43). Anchura máxima de la cápsula cefálica: 2.12$2.25 \mathrm{~mm}$. Superficie del cranium amarillo claro, sin puntos notables. Frente con una seda frontal exterior, y dos sedas frontales posteriores a cada lado, 2 sedas frontales anteriores, 1 seda en ángulos anteriores a la frente; con 2-4 sedas dorsoepicraneales, y 5-6 sedas paraocelares. Ocelos bien definidos. Clipeo con 2 sedas centrales y 2 sedas laterales a cada lado. Labro ligeramente asimétrico con 4-5 sedas largas posteriores, 2 sedas centrales y 3-4 sedas laterales a cada lado. La epifaringe (Fig. 44) tiene una anchura de $1.05-1.07 \mathrm{~mm}$ y una longitud de $0.68-0.80$, con epizygum alargado irregular pardo oscuro, haptomerum con 3-4 heli bien pronunciados, cada plegmatium formado con 7-7 plegmata poco visibles, acanthoparia con 6-7 sedas espiniformes cortas, corypha con 6 sedas, acroparia con 20 sedas, pedium amplio. Carece de proplegmatia, clithra y dexiophoba. Laeophoba grande, se extiende a través del borde de la laeotorma. Dexiotorma casi recta, laeotorma corta, ensanchada; placa esclerosada grande y angulosa, cono sensorial grande. Crepis poco marcado. Chaetoparia izquierda con 30 sedas gruesas y carece de sedas delgadas. Chaetoparia derecha con 21 sedas gruesas y carece de sedas delgadas. Gymnoparia amplia. Mandíbulas (Fig. 45-46) sin área estriduladora ventral. Áreas incisivas de ambas mandíbulas con una lámina cortante distal separada de un dientecillo incisivo proximal por una escotadura incisiva; márgenes internos sin dientes; área molar de la mandíbula izquierda con el lóbulo distal (M1) bien desarrollado; acia no desarrollada; brustia moderadamente 

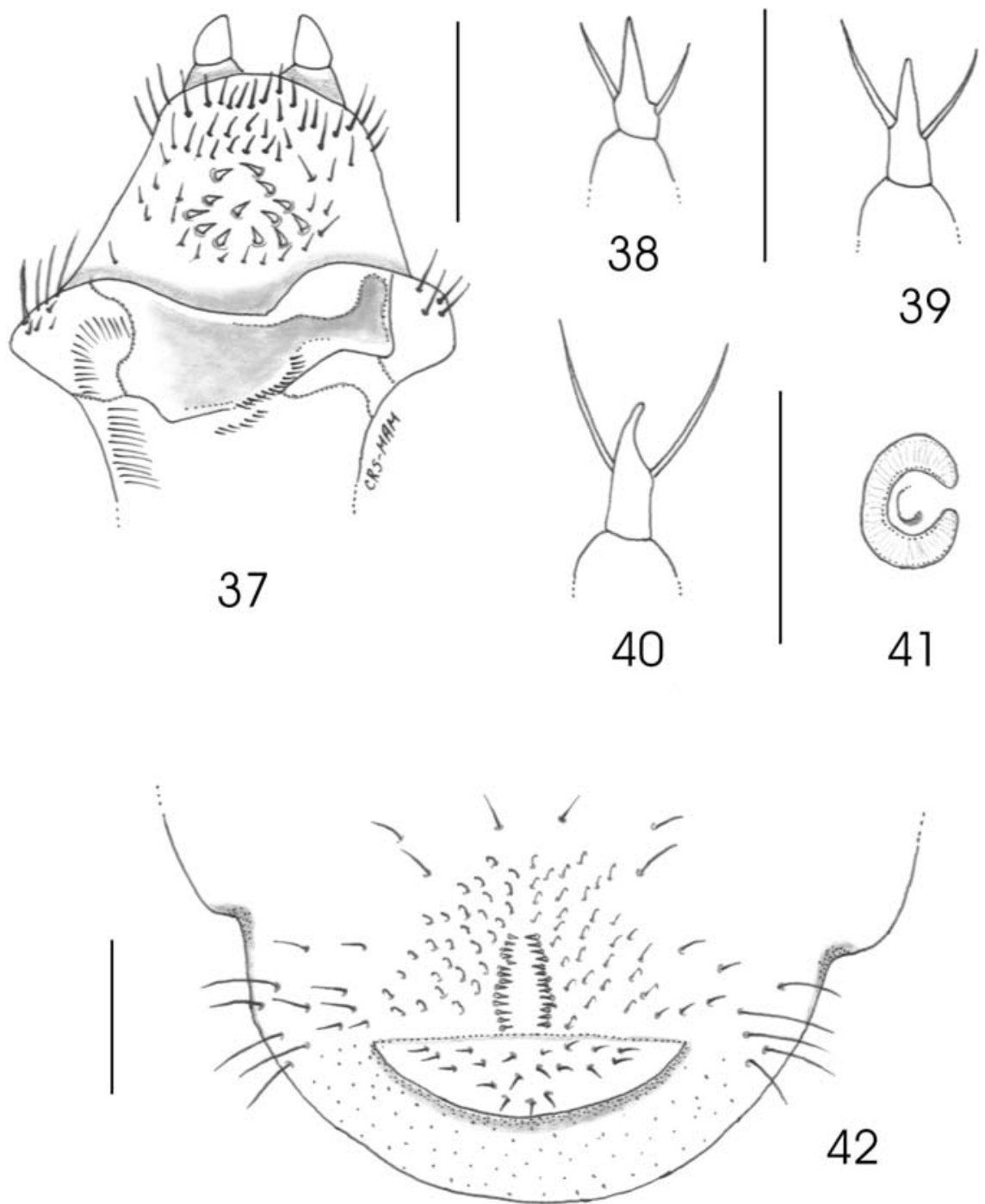

Figuras 37-42. Larva de tercer estadio de Paranomala trapezifera. 37. Hipofaringe. Uñas: 38. protarsal. 39. mesotarsal. 40. metatarsal. 41. Estigma respiratorio protorácico. 42. Raster. Líneas de escala $=0.5 \mathrm{~mm}$, excepto en fig. $42=1 \mathrm{~mm}$.

setosa; área molar de la mandíbula derecha con tres lóbulos poco diferenciados y brustia moderadamente setosa. Maxilas (Fig.47-48) con 1 uncus en la galea, 3 unci alineados en lacinia, siendo el intermedio más pequeño, y con hileras de 3 heli a cada lado. Área estriduladora maxilar formada por 8-10 dientecillos. Escleroma hipofa- 
ríngeo asimétrico (Fig. 51), con el lado derecho prominente y el izquierdo reducido. Último artejo antenal (Figs. 49-50) con una área sensorial ovalada en la superficie dorsal y dos áreas en la superficie ventral.

Tórax. Pronoto con un escleroma lateral corto a cada lado, blanco cremoso, rodeado de 8-15 sedas medianas y largas. Los estigmas respiratorios pequeños, blan-
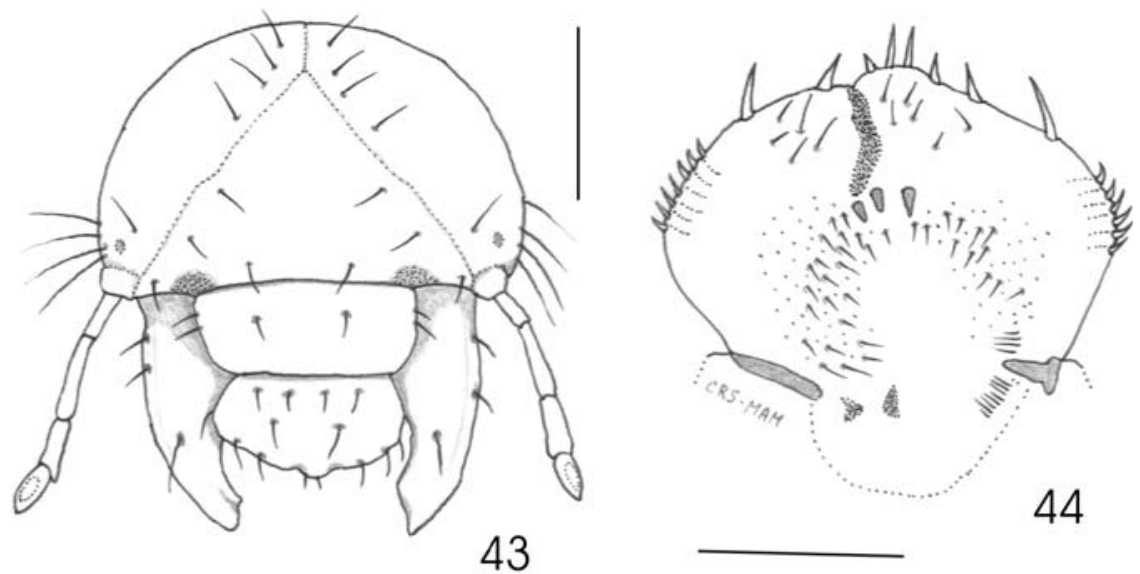

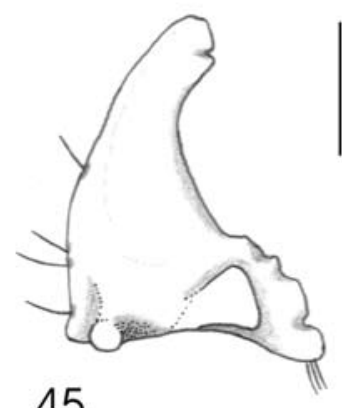

45

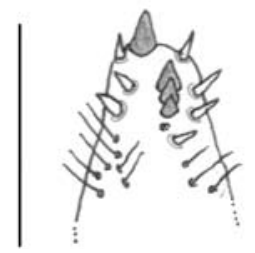

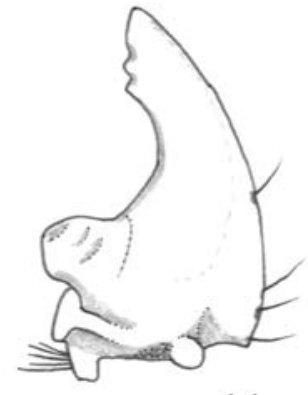

46

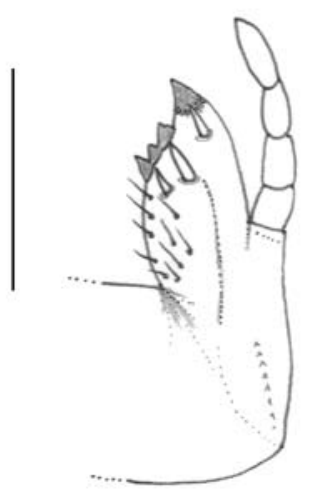

47

Figuras 43-50. Larva de tercer estadio de Macrodactylus fulvescens. 43. Vista frontal de la cabeza. 44. Epifaringe. Mandíbulas en vista ventral: 45. izquierda. 46. derecha. 47. Maxila derecha, dorsal. 48. Vista mesial del ápice de la maxila derecha. Último artejo antenal: 49. ventral. 50. dorsal. Líneas de escala $=0.5 \mathrm{~mm}$. 
quecinos, poco pronunciados. Proprescudo con 5-8 sedas cortas y largas distribuidas irregularmente, proescudo con 8-10 sedas largas en hilera transversal, mesoprescudo con 6-8 sedas largas transversales, mesoescudo sin sedas, mesoescutelo con 6-7 sedas medianas y largas en hilera tranversal, metaprescudo con 7-8 sedas largas en hilera tranversal, metaescudo sin sedas, metaescutelo con 8-9 sedas en hilera transversal. Todas las uñas tarsales (Fig. 52-54) casi con la misma forma y dimensiones, con una seda prebasal interna y una seda externa intermedia; pero las uñas metatarsales son un poco más cortas y recurvadas.

Abdomen. Estigmas de los segmentos I-VIII pequeños, blanquecinos, poco notables. Dorsa de los segmentos abdominales I-VI con numerosas sedas espiniformes y algunas sedas largas distribuidas transversalmente; segmentos VII-IX sin sedas espiniformes con hileras transversales de sedas largas. Venter de los segmentos I-IX con hileras transversas de sedas medianas y largas. Raster (Fig. 56) con un par de palidia corta y estrecha, con una longitud de 0.27-0.48 mm; cada palidium está formada por 4-7 pali. Tegilla con 20-22 sedas hamate. Campus con 10 sedas largas y finas. Barbula escasa. Labio anal dorsal con sedas largas. Labio anal ventral con sedas cortas esparcidas. Abertura anal en forma de "V". Longitud total dorsal del cuerpo aproximada: $2.4-2.5 \mathrm{~cm}$.

Comentarios. Las larvas de Macrodactylus fulvescens se diferencian de las otras especies estudiadas en Los Altos de Chiapas (Ramírez-Salinas et al. 2000, 2001, 2004) por su cuerpo más pequeño y esbelto, cabeza con ocelos bien definidos, estigmas respiratorios pequeños, blanquecinos, apenas visibles, un par de palidia cortas y estrechas, cada palidium formado por 4-7 pali, y una abertura anal en forma de "V".

Pupa macho (Fig. 57). $(\mathrm{N}=1)$. Longitud dorsal total del cuerpo: $10 \mathrm{~mm}$. Cabeza. Glabra, fuertemente inclinada hacia abajo; ojos, piezas bucales y tecae antenales claramente diferenciados.

Tórax. Pronoto un poco más largo que ancho, convexo; meso, metanoto y escutelo diferenciados, tecae elitrales y alares proporcionalmente cortas, recurvadas alrededor del tórax y la base del abdomen; fémures y tibias estrechamente plegados, tarsos más largos que las tibias, especialmente en las patas intermedias y posteriores.

Abdomen. Con nueve segmentos diferenciables; carece de órganos dioneiformes, pero en el dorso de los segmentos II a VI muestra quillas transversales prominentes; los estigmas respiratorios de los segmentos II a VI tienen un ostium circular pequeño, con peritrema fino, rodeado por una amplia fosa delimitada con un reborde ovalado irregular que se continúa con la quilla dorsal transversal de cada segmento. Los estigmas respiratorios de los segmentos VII y VIII son mas pequeños y poco distinguibles, carecen de la fosa y el reborde, pero están rodeados por rugosidades finas irregulares, no rosetiformes. El último segmento carece de urogomphi, pero se aprecian lóbulos 

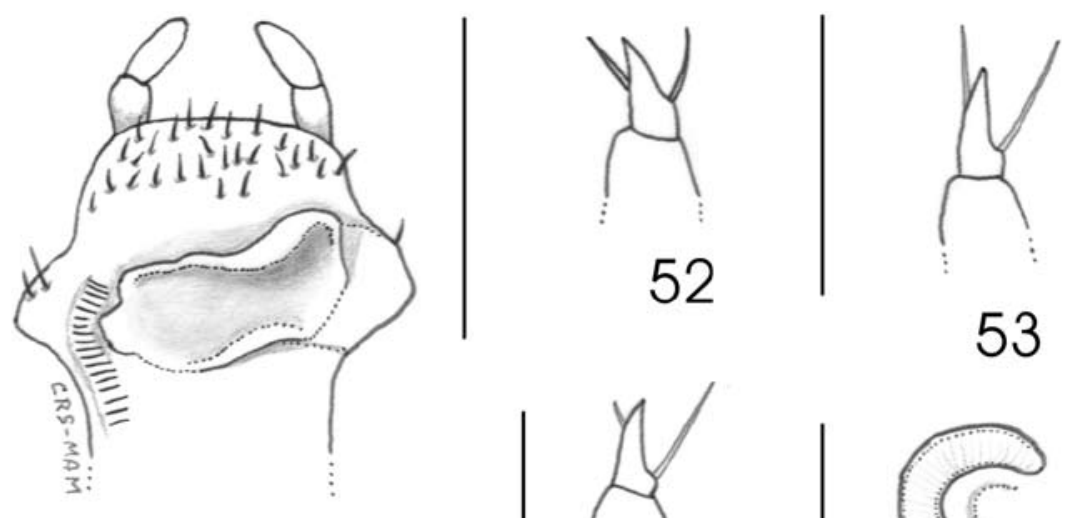

\section{1}
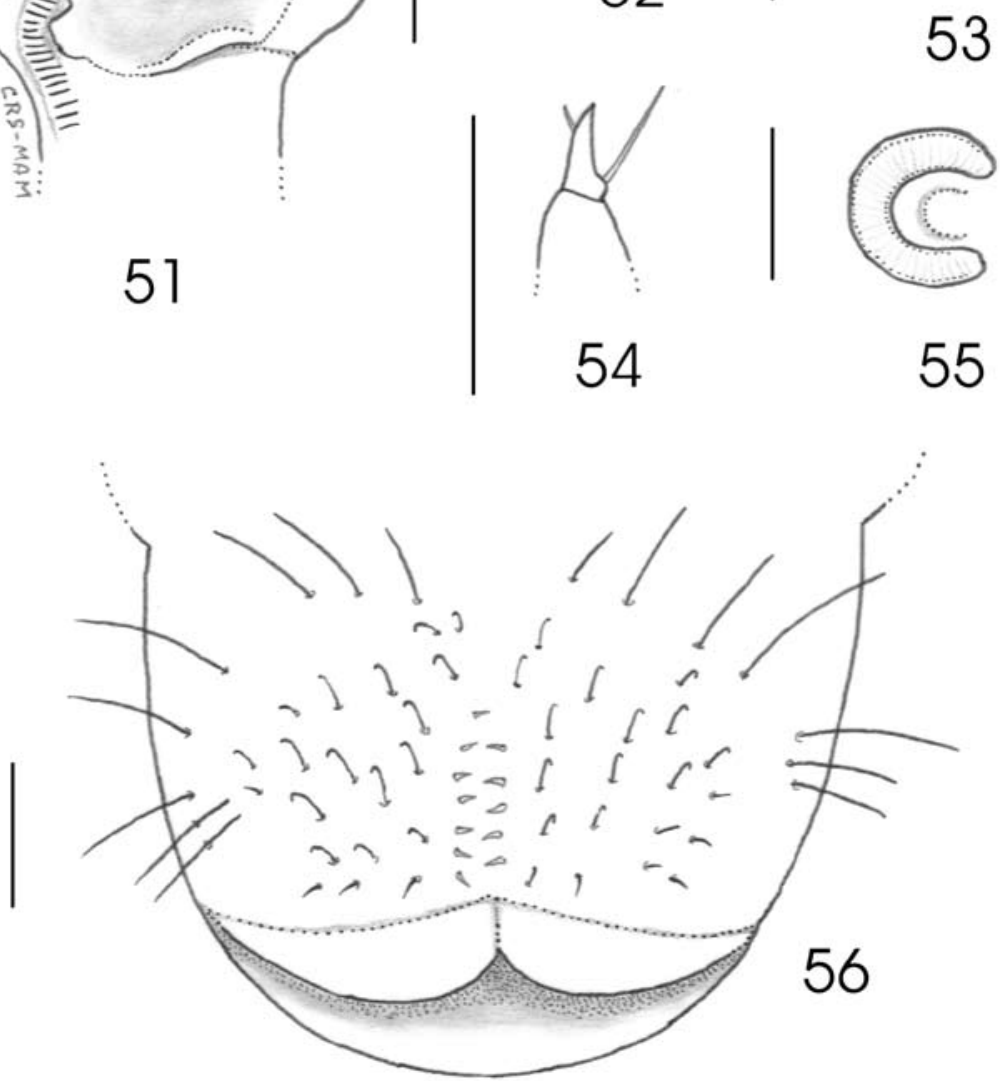

Figuras 51-56. Larva de tercer estadio de Macrodactylus fulvescens. 51. Hipofaringe. Uñas:

52. protarsal. 53. mesotarsal. 54. metatarsal. 55. Estigma respiratorio protorácico. 56. Raster. Líneas de escala $=0.5 \mathrm{~mm}$.

transversales dorso-apicales y una prominencia modesta ventro-apical que puede corresponder con una ámpula genital.

Material examinado. Una pupa de macho obtenida el 19-IV-2002 de la cría de larvas colectadas en campo el 10-IX-2001 en México: Chiapas, municipio Comitán, 


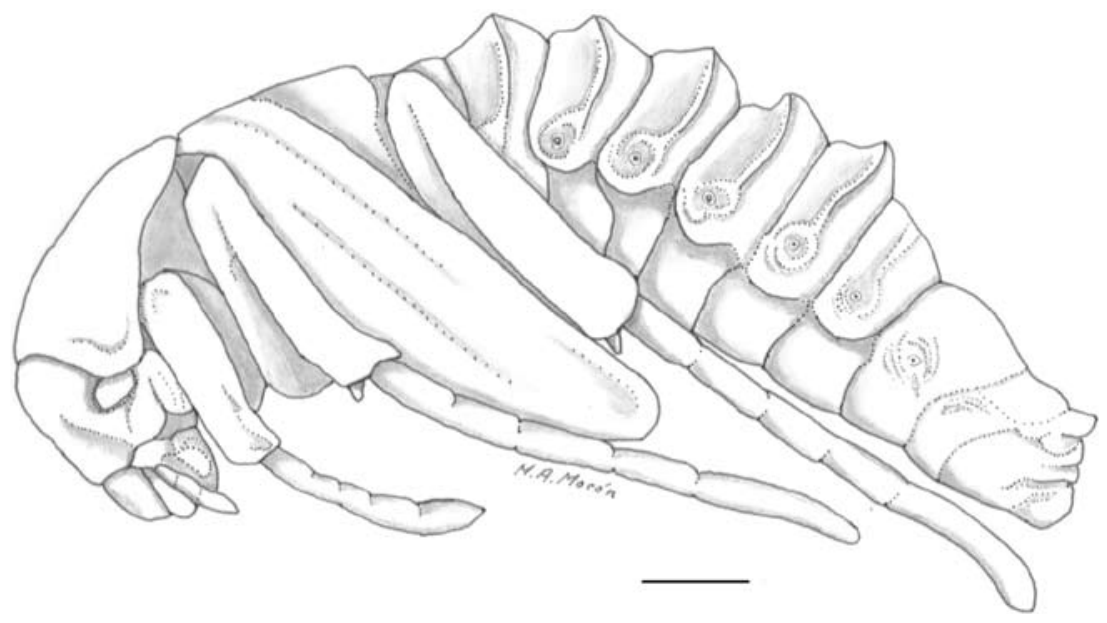

Figura 57. Vista lateral de la pupa de un macho de Macrodactylus fulvescens. Escala $=1 \mathrm{~mm}$.

comunidad Yalumá Villahermosa. Cultivo maíz. C. Ramírez Salinas y A. Castro Ramírez.

Comentarios. Es la primera vez que se describe e ilustra la pupa de una especie de Macrodactylus. A reserva de estudiar otras especies, una de las características notables para distinguir a las pupas de este género es la estructura dorsal del abdomen, con quillas transversales fuertes, en combinación con la forma y ubicación de los estigmas respiratorios, la ausencia de urogomphi y la gran longitud de los tarsos.

AGRADECIMIENTOS. Al Dr. Guillermo Ibarra Núñez, de ECOSUR Tapachula, por prestarnos el equipo para realizar las mediciones de las larvas, y al Ing. Jesús Carmona de la Torre por las recolectas de los escarabajos Phyllophaga pilosula y Paranomala trapezifera.

\section{LITERATURA CITADA}

Castro-Ramírez, A. E., C. Ramírez-Salinas \& C. Pacheco-Flores. 2004. Guía ilustrada sobre "gallina ciega" en la región Altos de Chiapas. El Colegio de la Frontera Sur y Benemérita Universidad Autónoma de Puebla. México. 48 p.

Castro-Ramírez, A. E., H. Delfín González, V. Parra Tabla \& M. A. Morón. 2005. Fauna de melolóntidos (Coleoptera: Scarabaeoidea) asociados al maíz (Zea mays L.) en los Altos de Chiapas, México. Folia Entomológica Mexicana, 44(3): 339-365.

Morón, M. A. 1986. El género Phyllophaga en México. Morfología, distribución y sistemática supraespecífica (Insecta: Coleoptera). Pub.19. Instituto de Ecología. México. 344 pp.

Morón, M. A., B.C. Ratcliffe \& C. Deloya. 1997. Atlas de los escarabajos de México. Coleoptera Lamellicornia. Vol. I. Familia Melolonthidae. CONABIO y Sociedad Mexicana de Entomología, México. 280 pp. 
Boving, A. 1942. A classification of larvae and adults of the genus Phyllophaga (Coleoptera: Scarabaeidae). Memoirs of the Entomological Society of Washington, 2: 1-95.

Ramírez-Salinas, C., M. A. Morón \& A. E. Castro-Ramírez. 2000. Descripción de los estados inmaduros de seis especies de Phyllophaga (Coleoptera: Melolonthidae; Melolonthinae) de la región Altos de Chiapas, México. Folia Entomológica Mexicana, 109: 73-106.

Ramírez-Salinas, C., A. E. Castro-Ramírez \& M. A. Morón. 2001. Descripción de la larva y pupa de Euphoria basalis (Gory y Percheron, 1833) (Coleoptera: Melolonthidae: Cetoniinae) con observaciones sobre su biología. Acta Zoológica Mexicana (n.s.), 83: 73-82.

Ramírez-Salinas, C., M. A. Morón \& A. E. Castro-Ramírez. 2004. Descripción de los estados inmaduros de tres especies de Anomala, Ancognatha y Ligyrus (Coleoptera: Melolonthidae: Rutelinae y Dynastinae) con observaciones de su biología. Acta Zoológica Mexicana (n.s.), 20(3): 67-82.

Ritcher, P. O. 1966. White grubs and their allies: a study of North American Scarabaeoid larvae. Studies in Entomology No. 4, Oregon State University Press, Corvallis. 219 pp. 